\title{
Crowd evacuation simulation for walking-along-side effect in the Stadium
}

\author{
Benbu Liang ${ }^{1, *}$, Kefan Xie ${ }^{1}$, and Xueqin Dong ${ }^{1}$ \\ ${ }^{1}$ School of Management, Wuhan University of Technology, Wuhan, P.R. China
}

Keywords: Crowd evacuation, Simulation, Walking-along-side effect, Stadium.

\begin{abstract}
With growing concerns about stadiums where attract large mass gathering, modeling and simulating crowd evacuation is pertinent to ensuring efficient and safe conditions. Based on the modified social force model and multi-agent simulation, several simulation scenarios are conducted to study the walking-along-side effects. The results show that walking along the sides will increase evacuation time, but it can mitigate the pressure of clogging effects and stream arching queue. Meanwhile, walking-along-side effects can relieve the density pressure of the exit and the "fast-is-slow" phenomenon. At last, several suggestions are put forward to promote evacuating capacity of the stadium.
\end{abstract}

\section{Introduction}

In recent years, with increasing demands for entertainment and social communication, mass gatherings are becoming even more frequency [1]. As a consequence, there are many situations in which thousands of people gather. Especially more music and sport events with a large number of spectators are taking place in kinds of stadiums. On August 22,2019, the crowd stampede at a concert in Algeria, where 24 people injured and 5 people died, illustrates the need for developing safety crowd evacuation management and appropriate stadium design.

The study of crowd evacuation and simulation has aroused great attention, due to the flexibility of a large amount of pedestrians and the complex nonlinear interaction between them, and the external factors such as the topographic characteristics of the environment [2], the layout of a pedestrian facility of stadiums [3]. Previous studies have approved that modeling and simulation are effective to program crow evacuation [4]. Generally, these models are classified into two categories (i.e., macroscopic and microscopic model) which are widely conducted to analyze crowd evacuation rules in stadium. Macroscopic models regard the crowd as a whole, and explore the expression of fundamental diagram [5]. Microscopic models focus on qualitatively explaining the evacuation dynamics, including spatial-discrete models. In addition, the density, flow or crowd pressure of crowding motion

\footnotetext{
*Corresponding author: liangbenbu@163.com
} 
plays a significant role on crowd evacuation [6]. Although demographics and anthropometry of pedestrians influenced the evacuation time, the stadium architecture characteristics were the main factor which affected pedestrian flow [7]. Liao et al.[8] had concluded that the layout of ticket-inspectors has significant effects on evacuation efficiency. Zhang et al.[9] had proposed a stranded-crowd model to predict the relationship between the stadium egress width and stranded number of panic crowd. Besides, walking-along-side effect, imitation effect and non-optimal effect was tested in out-door public places [10]. Other researchers investigated how various factors including exists visibility, physical conditions of exits related to the pedestrians' exit choice [11].

Previous studies on mass gathering or evacuation within stadiums can be classified into two different situation based on modelling techniques (i.e. modelling techniques used in descriptive models and optimization models), which analyzed issues involved crowd evacuation rout choice and venue layout from a macro perspective. However, most of these models ignored the micro-level detail. Example like that pedestrians prefer to walking along side of the wall particularly when they in an unfamiliar environment. Even the well-known social force model (SFM), although reproducing several self-organized phenomena, it assumes that there is always repulsion between the pedestrian and the wall. This study proposes a model based on the improved SFM and multi-agent method to analyze the walking-along-side effect in a simplified stadium. The paper is organized as follows: the next section develops an improved SFM coupled with the simulation scenarios and protocol parameters of the stadium. The corresponding simulation scenarios are conducted and simulation results are presented in Section 3. Section 4 summarizes the main results of simulation and suggestions.

\section{Methodology}

\subsection{Formulation of simulation model}

The crowd is a complicated system full of interactions between pedestrian-pedestrian and pedestrian-environment in the process of evacuation [12]. Based on Newton's second law, SFM regards pedestrian as self-driven multi-particles. Usually pedestrian $i$ will be subject to self-drive $f_{i}$, pedestrian repulsion $f_{i j}$ and obstacle repulsion $f_{i w}$ constraints. In order to enhance the traditional SFM, the preference of pedestrian-environment is involved. The social force of the individual is defined as the resultant force of repulsive or attractive force generated by environmental preference. Let

$$
m_{i} \frac{d v_{i}}{d t}=\left(1+\lambda E_{i}^{t}\right) f_{i}\left(v_{i}, v_{i}^{0} e_{i}^{0}\right)+\sum_{i \neq j)} f_{i j}\left(e_{i}, r_{i}-r_{j}\right)+\left(\lambda E_{i}^{t}\right) \sum_{w} f_{i w}\left(e_{i}, r_{i}-r_{w}\right)+\xi \text { (1) }
$$

where $m_{i}$ represents the mass of pedestrian $i, v_{i}$ is his or her expected speed. $f_{i}, f_{i j}, f_{i w}$ respectively denote the different dimension of social force. $\lambda$ means the preference of pedestrian-environment while $\xi$ represents random pedestrian disturbance term. Then, the Eq. 1 can be written with details as:

$$
f_{i}\left(v_{i}, v_{i}^{0} e_{i}^{0}\right)=m_{i} \frac{v_{i}^{0}(t) e_{i}^{0}(t)-v_{i}(t)}{\tau_{\mathrm{i}}},
$$




$$
\begin{gathered}
f_{i j}\left(e_{i}, r_{i}-r_{j}\right)=\mathrm{A}_{i} \exp \left(\frac{r_{i j}-d_{i j}}{B_{i}}\right) n_{i j}+k \Theta\left(r_{i j}-d_{i j}\right) n_{i j}+K \Theta\left(r_{i j}-d_{i j}\right) \Delta v_{i j}(t), \\
f_{i \mathrm{w}}\left(e_{i}, r_{i}-r_{w}\right)=\mathrm{A}_{i} \exp \left(\frac{r_{i}-\mu^{-1} d_{i w}}{B_{i}}\right)+k \Theta\left(r_{i}-\mu^{-1} d_{i w}\right)+K \Theta\left(r_{i}-d_{i w}\right) \Delta v_{i w}(t),
\end{gathered}
$$

The parameter $\mu(0<\mu \leq 1)$ denotes the sensitivity coefficient of pedestrians to the distance between obstacles. When $\mu$ is smaller, it indicates that pedestrians have less environmental preference. Where $A_{i}, B_{i}, k, K$ constant while $d_{i j}, d_{i w}$ represent the distance between pedestrian-pedestrian and pedestrian-environment. Notice that when $r-d<0$, $\Theta(x)=0$, otherwise, $\Theta(x)=r-d$.

\subsection{Scenario constructions}

Taking a stadium in Wuhan China as the research object, simplified stadium model depending on the investigation and measurement (Fig. 1). The simplified stadium model is mainly divided into the main body area $(65 \mathrm{~m} \times 40 \mathrm{~m})$, audience area $(65 \mathrm{~m} \times 10 \mathrm{~m})$ and performance area $(30 \mathrm{~m} \times 20 \mathrm{~m})$. There is only one-single exit $(3 \mathrm{~m})$, right in the middle of the wall facing the audience area. When an emergency occurs, pedestrians will escape from the audience area, cross the main body area, and leave from the exit. According to the calculation, there are at least 2,000 seats in the audience area, and each position is connected into a seats column, forming an obstacle.

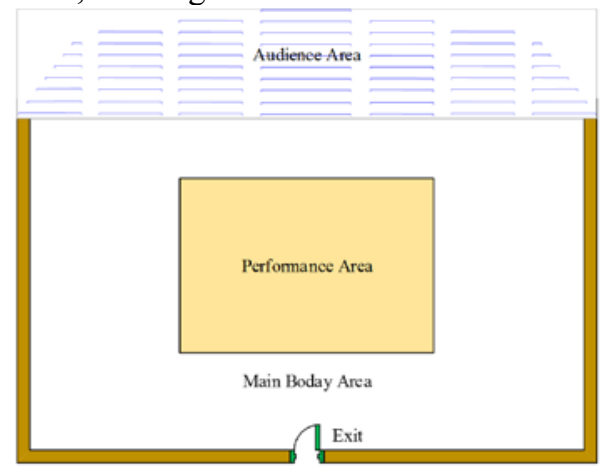

(a)

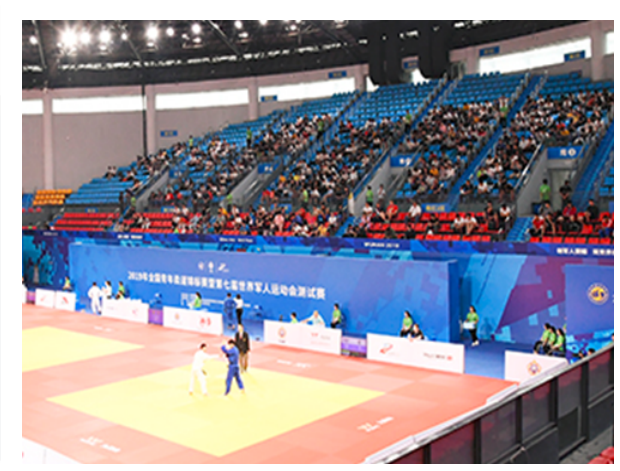

(b)

Fig. 1. (a) Sketch of the layout in the stadium; (b) snapshot of the stadium in reality.

Combined with the observation, assuming that comfortable speed of pedestrians obey $N(0.8,0.01)(\mathrm{m} / \mathrm{s})$, and initial speed obey $U(0.3,0.7)(\mathrm{m} / \mathrm{s})$. Due to the individual difference, the shoulder width obey $U(0.35,0.5)(\mathrm{m})$. Considering with walking-along-side effect, three simulation scenarios (SS) are designed (shown in Table 1). SS1 routine on normal evacuation like pedestrians looking for the shortest path to escape. In SS 2, considering a strong preference for walls, all the pedestrians should evacuate along the sides of the stadium. The number of pedestrians in SS1 and SS2 increases from 500 by 300 to 2000. SS3 considers the heterogeneity of pedestrians' preference for the wall, the proportion of 2000 pedestrians 
choosing to walking-along-side increased $20 \%$ from $10 \%$ to $100 \%$. Note that N/W which means the ratio of the number of pedestrians looking for the nearest exit to pedestrians walking along the sides. In addition, in order to increase the stability of the simulation results, every SS repeat for 5 times to calculate the mean value as the final simulation result.

\section{Result analysis and discussion}

\subsection{Evacuation time}

Safe evacuation time is the most direct evidence to evaluate an evacuation model. According to Fig. 2, the evacuation time in SS1 is always less than SS2 under the same number of pedestrians, showing that it's not effectively when pedestrians all walking along the sides. Moreover, evacuation time in SS3 all longer than 6 sub-SS1, and most of the sub-SS3s such as SS3-2 (N/W=3:2), SS3-3 $(\mathrm{N} / \mathrm{W}=1: 1)$ and SS3-5 $(\mathrm{N} / \mathrm{W}=1: 4)$ are efficient than $\mathrm{SS} 2$. Although edging slows down the crowding effect of exits, it generally takes longer because pedestrians have to walk farther to reach the exits. However, the walking-along-side effect is not so sensitive as the selection of the optimal route when the number of pedestrians increasing. In Figure. 2, the variance of curve SS2 is smaller than that of SS1, because pedestrians can have a larger spatial buffer beside walls or corners.

\subsection{Density map}

Overcrowding is the direct cause of stampede. Therefore, the analysis of the density map generated by simulation will be helpful to understand the potential risk points in the whole evacuation. According to previous studies, the crowd density threshold defined for stampede is 5.26 person $/ \mathrm{m}^{2}$, which is conducted at the highest density set in this paper. Fig. 3 shows 4 density maps of different is sub-SSs, where (a) is SS1-6, (b) is SS3-2, (c) is SS3-4 and (d) is SS2-6. Obviously, from Fig. 3, we can see that the density map of crowd evacuation at a single exit is funnel-shaped, and the friction between pedestrians at the only exit leads to "fast-is-slow" phenomenon. With the decrease of N/W value, the arch queue effect at the exit is less obvious. Therefore, although the logging effects cannot be effectively solved, the walking-along-side effects will shift the dynamic bottleneck to the corner. Hence, it will greatly reduce the probability of the crowd trample density.

\subsection{Pedestrian flow}

Similar to density map, pedestrian flow which means the number of pedestrian passing per unit of time, can be used to monitor the hazardous situation of mass gathering as well. As shown in Fig. 4, it can be noted that, in the evacuation process within the same number of population, the maximum and the mean value of pedestrian flow in SS2 are always smaller than SS1, which indirectly indicates that the walking-along-side effects can effectively relieve the pressure of the exit. In addition, the lower quartile in all SSs box diagrams coincides with the lower limit (i.e. 0), which represents that there exists serious clogging effects to generate crowds intermittent. Particularly, when the number of pedestrians in less (less than 1400), the mean value is greater than the median value in the box diagrams, indicating the distribution of pedestrian flow in the right condition. On the contrary, with the increasing of the population in the process of evacuation, the median value start beyond the 
mean value, indicating that the distribution turn to leave, which shows that low efficiency of clogging effects seriously hindered the evacuation process.

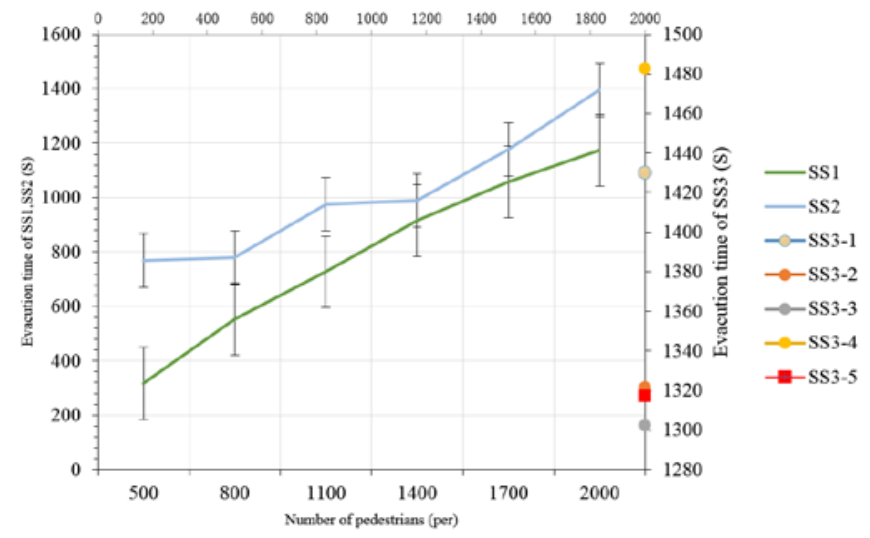

Fig. 2. The evacuation time under different number of pedestrians in SS1 3.

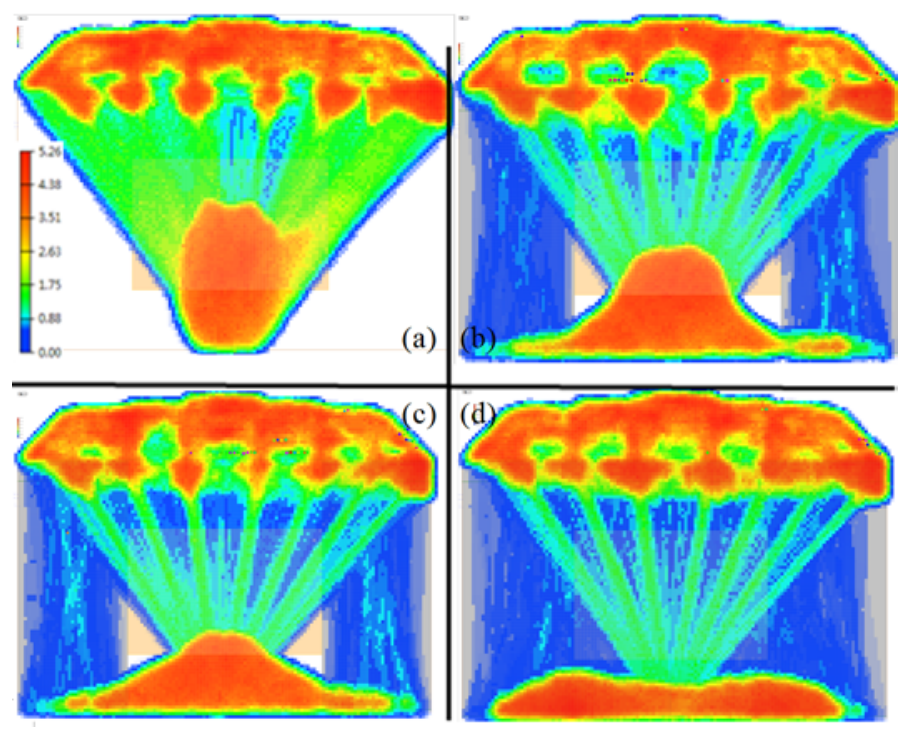

Fig. 3. The density maps of 4 typical sub-SSs. 


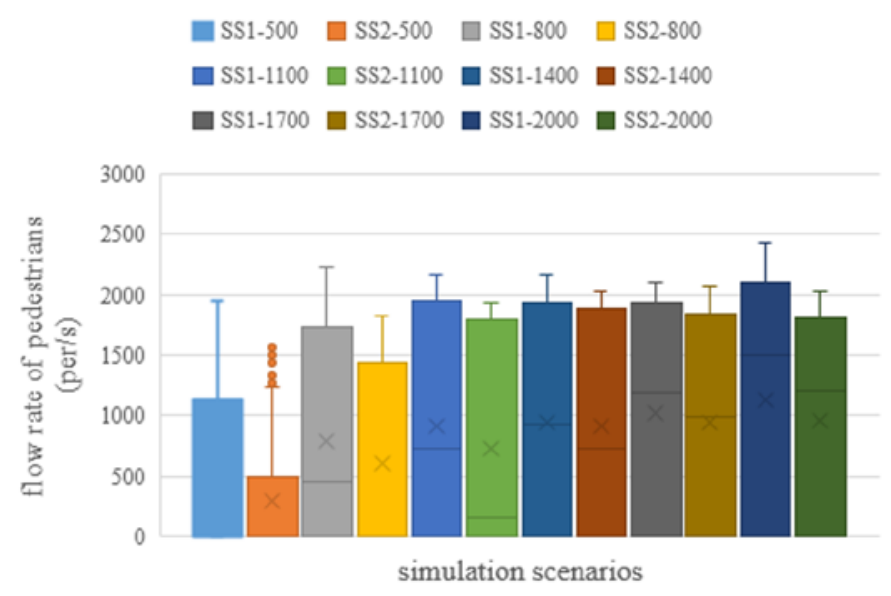

Fig. 4. Box diagrams of pedestrian flow in SS1 and SS2.

\section{Summary}

This study explored the crowd evacuation in stadium based on the modified SFM and multi-agent simulation. Several simulation scenarios were conducted to analyze the walking-along-side effects. In the simulation, all pedestrians escaped from a simplified stadium, and evacuation time, density map and pedestrian flow were recorded to explore patterns of crowd evacuation. It is noted that although walking-along-side would cost more time to evacuate, it can share the pressure of clogging effects and stream arching queue, thus reducing the risk of a stampede. Meanwhile, walking-along-side effects can relieve the density pressure of the exit and the "fast-is-slow" phenomenon.

Based on the above analysis, several suggestions and implications are given as follows: (1) avoid setting up one-single exit; (2) the key positions (e.g. entrances, exits and corridors) in stadium should be monitored during the peak period of mass gathering; (3) for some entrance and exits which easily attract a large number people, signs should be set up to remind pedestrians walking along the sides or detour; (4) for large stadium, apart from the main venue, sufficient space should be built to ensure that pedestrians can walk along the sides, hence reducing clogging effects and avoiding arching queue.

However, it should be pointed that the stadium model is simplified. Moreover, the SSs selection is not utterly objective. Therefore, problems about the layout of the stadium and the mechanism of walking-along-side effects should be studied in future work.

This research was financially supported by Manufacturing Industry Development Research Center on Wuhan City Cycle, Jianghan University.

\section{References}

1. De Almeida M M, von Schreeb J. Human stampedes: an updated review of current literature[J]. Prehospital and disaster medicine, 2019,34(1):82-88.

2. Li, Wang, Bo, et al. Incorporating topography in a cellular automata model to simulate;residents evacuation in a mountain area in China[J]. Physica A Statistical Mechanics \& Its Applications, 2013,392(3):520-528. 
3. Wu Y, Kang J, Wang C. A crowd route choice evacuation model in large indoor building spaces[J]. Frontiers of Architectural Research, 2018,7(2):135-150.

4. Kefan X, Song Y, Liu S, et al. Analysis of crowd stampede risk mechanism: A systems thinking perspective[J]. Kybernetes, 2019,48(1):124-142.

5. Haghani M, Sarvi M. Crowd behaviour and motion: Empirical methods[J]. Transportation Research Part B Methodological, 2017,107:S1966321684.

6. Feliciani C, Nishinari K. Measurement of congestion and intrinsic risk in pedestrian crowds[J]. Transportation Research Part C Emerging Technologies, 2018,91:124-155.

7. Aucoin D R. The Use of Human Behaviour to Inform Egress Modeling in Stadiums[J]. 2019.

8. Liao W, Zheng X, Cheng L, et al. Layout effects of multi-exit ticket-inspectors on pedestrian evacuation[J]. Safety Science, 2014,70:1-8.

9. Zhang Q, Liu M, Caihong W U, et al. A stranded-crowd model (SCM) for performance-based design of stadium egress[J]. Building \& Environment, 2007,42(7):2630-2636.

10. Liu S S, Liu J, Wei W. Simulation of crowd evacuation behaviour in outdoor public places-a model based on shanghai stampede[J]. International Journal of Simulation Modelling, 2019,18(1): 86-99.

11. Ren-Yong G, Hai-Jun H. Logit-based exit choice model of evacuation in rooms with internal obstacles and multiple exits[J]. Chinese physics B, 2010,19(3):30501.

12. Helbing D, Farkas I, Vicsek T. Simulating dynamical features of escape panic[J]. Nature, 2000,407(6803):487. 